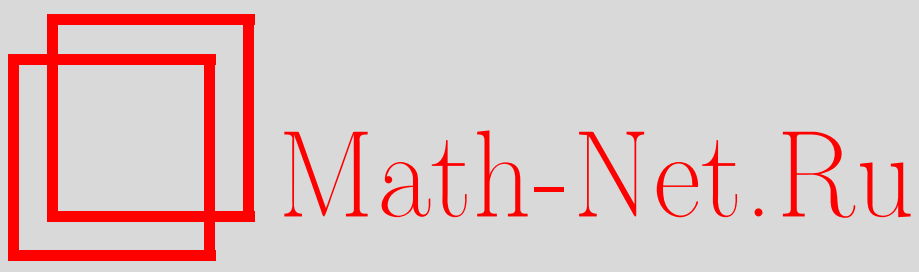

В. В. Беняш-Кривец, О разложении свободного произведения циклических групп с одним соотношением в амальгамированное свободное произведение, Матем. сб., 1998, том 189, номер 8, 13-26

DOI: https://doi.org/10.4213/sm332

Использование Общероссийского математического портала Math-Net.Ru подразумевает, что вы прочитали и согласны с пользовательским соглашением

http://www. mathnet.ru/rus/agreement

Параметры загрузки:

IP : 107.22 .136 .117

26 апреля 2023 г., 16:51:13 
УДК 512.543 .76

\author{
В.В. Беняш-Кривец
}

\title{
О разложении свободного произведения цик лических групп с одним соотношением в амальгамированное свободное произведение
}

\footnotetext{
Данная работа посвящена изучению проблемы разложения свободного произведения циклических групп с одним соотношением в нетривиалњное амалггамированное свободное произведение. Доказаны две теоремы, из которых отметим следующую.

Пусть $G=\left\langle a, b \mid a^{2 n}=R^{m}(a, b)=1\right\rangle$, әде $n \geqslant 0, m \geqslant 2, R(a, b)-$ ичклически редучированное слово в свободной группе, порожсднной а и $b$, которое содержит $b$. Тогда $G$ является нетривиальным амальгамированным свободным произведением.

В качестве следствия этой теоремы получаем доказательство гипотезы Файна, Левина и Розенбергера о том, что любая группа с двумя образующими и одним соотношением с кручением является нетривиальньм амальгамированньм свободным произведением.

Библиографйя: 13 названий.
}

\section{Введение}

Свободным произведением семейства групn $\left\{G_{i}\right\}, i \in I$, с одним соотношением назьвается группа $G=\left(* G_{i}\right) / N(S)$, где $S$ - циклически редуцированное слово в свободном произведении $* G_{i}, N(S)$ - его нормальное замыкание; $S$ называется соотношением. Свободные произведения с одним соотношением имеют много обших свойств с группами с одним соотношением [1]. Мы рассматриваем случай, когда $G_{i}$ является циклической группой и соотношение является собственной степенью, т.е. $S=R^{m}$, где $R$ - циклически редуцированное слово в $* G_{i}$ и $m \geqslant 2$.

ОпРЕДЕЛЕНИЕ 1. Группа $G$, имеющая копредставление

$$
G=\left\langle a_{1}, \ldots, a_{n} \mid a_{1}^{l_{1}}=\cdots=a_{n}^{l_{n}}=R^{m}\left(a_{1}, \ldots, a_{n}\right)=1\right\rangle,
$$

где $n \geqslant 2, m \geqslant 2, l_{i}=0$ или $l_{i} \geqslant 2$ для всех $i, R\left(a_{1}, \ldots, a_{n}\right)$ - циклически редуцированное слово в свободной группе, порожденной $a_{1}, \ldots, a_{n}$, называется свободным произведением $n$ ииклических групп с одним соотношением (опе relator product of cyclics в англоязычной литературе).

Работа вьполнена при финансовой поддержке Института математики НАН Беларуси в рамках государственной программы фундаментальыых исследований "Математические структуры". 
В данной работе рассматривается проблема разложения свободных произведений циклических групп с одним соотношением в нетривиальное амальгамированное свободное произведение. Первый общий результат о разложении таких групп был получен в [2]. Теорема 3 из [2] утверждает, что если $G$ является свободным произведением с одним соотношением $n$ циклических групп, $n \geqslant 3$, то $G$ является нетривиальным амальгамированным свободным произведением.

Случай свободного произведения двух циклических групп с одним соотношением, т.е. групп вида

$$
G=\left\langle a, b \mid a^{m}=b^{n}=R^{l}(a, b)=1\right\rangle,
$$

где $l \geqslant 2, R(a, b)$ - циклически редуцированное слово в свободной группе, порожденной $a$ и $b$, является значительно более сложным. Группы такого вида называют обобщенными треугольными группами. Эти группы иногда допускают разложение в нетривиальное амальгамированное свободное произведение, а иногда нет. Например, известно (см. [3]), что обычные треугольные группы

$$
T(m, n, p)=\left\langle a, b \mid a^{m}=b^{n}=(a b)^{p}=1\right\rangle,
$$

где $m, n, p \geqslant 2$, неразложимы в нетривиальное амальгамированное свободное произведение. В [3] Цишанг исследовал проблему разложения в амальгамированное свободное произведение для плоских разрывных групп. Он дал полньй ответ, когда такая группа является нетривиальньм амальгамированным свободным произведением, за исключением групп $H_{1}=\left\langle a, b \mid[a, b]^{n}=1\right\rangle$ и $H_{2}=\left\langle c, d \mid c^{2}=[c, d]^{n}=1\right\rangle$, $n \geqslant 2$. Розенбергер [4] доказал, что группы $H_{1}$ и $H_{2}$ являются нетривиальным амальгамированным свободным произведением в случае, когда $n$ не является степенью 2. В недавних работах [5], [6] этот факт доказан для произвольного $n$. Отметим, что этот результат является также непосредственным следствием теоремы 1. Следующая гипотеза высказана в [2]:

ГИПоТЕЗА 1. Произвольная группа с двумя образующими и одним соотношением с кручением является нетривиальным амальгамированным свободным произведением.

Отметим, что если группа $G$ имеет более двух образующих, то утверждение гипотезы 1 справедливо в силу упоминавшейся выше теоремы 3 из [2]. Кроме того, в [2] эта гипотеза доказана в случае, когда группа $G$ имеет копредставление $G=\left\langle a, b \mid R^{m}(a, b)=1\right\rangle$, где $m \geqslant 2$ и $R(a, b)$ - циклически редуцированное слово, не лежащее в коммутанте свободной группы, порожденной $a$ и $b$.

В предлагаемой работе доказываются следующие две теоремы.

Tеорема 1. Пусть $G=\left\langle a, b \mid a^{2 n}=R^{m}(a, b)=1\right\rangle$, дде $n \geqslant 0, m \geqslant 2, R(a, b)$ - ииклически редуцированное слово в свободной группе, порожсденной $а$ и $b$, которое содержит $b$. Тогда $G$ является нетривиальным амальгамированнылм свободным произведением.

Tеорема 2. Пусть $G=\left\langle a, b \mid a^{n}=R^{m}(a, b)=1\right\rangle$, где $n=0$ или $n \geqslant 2$, $m \geqslant 3$ и $R(a, b)$ - ииклически редуцированное слово в свободной группе с образуюшими а и $b$, содержащее $b$. Eсли $R(a, b)=a^{u_{1}} b^{v_{1}} \cdots a^{u_{s}} b^{v_{s}}$, где $0<u_{i}<n$, $v_{i} \neq 0$ для $i=1, \ldots, s$ u $\prod_{i=1}^{s}\left|v_{i}\right| \geqslant 3$, то $G$ является нетривиальным амальгамированным свободным произведением. 
СЛЕДСТВИЕ 1. Пусть $G$ - группа с двумя образующими и одним соотношением с кручением. Тогда $G$ является нетривиальным амальгамированным свободньм произведением.

ДокАЗАТЕЛЬСТво СЛЕДСТвИЯ 1. Хорошо известно, (см., например, [7]), что если $G$ - конечно порожденная группа с одним соотношением с кручением, то $G$ имеет копредставление вида $G=\left\langle a_{1}, \ldots, a_{n} \mid R^{m}\left(a_{1}, \ldots, a_{n}\right)=1\right\rangle$, где $m \geqslant 2$, $R\left(a_{1}, \ldots, a_{n}\right)$ - циклически редуцированное слово в свободной группе, порожденной $a_{1}, \ldots, a_{n}$. В нашем случае $G=\left\langle a, b \mid R^{m}(a, b)=1\right\rangle$ и согласно теореме 2 $G$ является нетривиальным амальгамированным свободным произведением.

СлеДСТВИе 2. Группь $H_{1}=\left\langle a, b \mid[a, b]^{n}=1\right\rangle$ u $H_{2}=\left\langle c, d \mid c^{2}=[c, d]^{n}=1\right\rangle$ являются нетривиальным амальгамированным свободныцм произведением для любого $n \geqslant 2$.

\section{§1. Некоторые вспомогательные результаты}

Здесь мы докажем ряд вспомогательных утверждений. Далее через $E$ мы будем обозначать единичную матрицу в $\mathrm{SL}_{2}(\mathbb{C}), \mathscr{O}$ - кольцо цельх алгебраических чисел в $\mathbb{C}, F_{2}=\langle g, h\rangle$ - свободную групшу ранга 2 с образующими $g$ и $h, \operatorname{tr} X-$ след матрицы $X$. Следуюший результат Басса [8] играет ключевую роль в доказательстве теорем 1 и 2.

ПРЕДЛОЖЕНИЕ 1 (см. [8]). Пусть $H$ - конечно порожденная подгруппа $\mathrm{GL}_{2}(\mathbb{C})$. Тогда имеет место один из следующих случаев:

1) существует әпиморфизм $f: H \rightarrow \mathbb{Z}$ такой, что $f(u)=0$ для всех унипотентных әлементов $u \in H$;

2) Н сопряжена подгруппе группы треугольных матрии вида $\left(\begin{array}{ll}a & b \\ 0 & c\end{array}\right)$, где а и с-корни из единицы;

3) $\mathrm{H}$ сопряжена подгруппе группь $\mathrm{GL}_{2}(\mathscr{O})$;

4) Н является нетривиальным амальгамированным свободным произведением.

Следуюшее наблюдение полезно для построения матрищ конечного порядка в $\mathrm{SL}_{2}(\mathbb{C})$.

Лемма 1. Пусть $m>1 u X \in \mathrm{SL}_{2}(\mathbb{C}) . \quad$ Если $\operatorname{tr} X= \pm 2 \cos (r \pi / m)$, əде $r \in\{1, \ldots, m-1\}$, mо $X^{2 m}=E$. В частности, если $\operatorname{tr} X=0$, mо $X^{2}=-E$.

Доказательство. Пусть, например, $\operatorname{tr} X=2 \cos (r \pi / m)$. Тогда характеристический многочлен матрицы $X$ имеет корни $\alpha=\cos (r \pi / m)+i \sin (r \pi / m)$ и $\alpha^{-1}=\cos (r \pi / m)-i \sin (r \pi / m)$, где $\alpha$ является корнем степени $2 m$ из 1 . Таким образом, $X$ сопряжена матрице $X_{1}=\operatorname{diag}\left(\alpha, \alpha^{-1}\right)$, следовательно, $X^{2 m}=E$.

Далее нам потребуются так называемые "характеры Фрике" (см. [9]-[11]). Для произвольного элемента $w=w(g, h) \in F_{2}$ рассмотрим следуюшую регулярную функцию:

$$
\tau_{w}: \mathrm{SL}_{2}(\mathbb{C}) \times \mathrm{SL}_{2}(\mathbb{C}) \rightarrow \mathbb{C}, \quad \tau_{w}(A, B)=\operatorname{tr}(w(A, B))
$$


В [9] доказано (см. также [12]), что для произвольного $w \in F_{2}$

$$
\tau_{w}=Q_{w}\left(\tau_{g}, \tau_{h}, \tau_{g h}\right)
$$

где $Q_{w} \in \mathbb{Z}[x, y, z]$ - многочлен с целыми коэффициентами. Функцию $\tau_{w}$ обычно называют характером Фрике, а многочлен $Q_{w}$ - многочленом Фрике элемента $w \in F_{2}$. Пусть $u, v$ - произвольные элементы $F_{2}$. Следуюшие соотношения между характерами Фрике следуют из соотношений между следами произвольных матриц в $\mathrm{SL}_{2}(\mathbb{C})$ и могут быть легко проверены:

$$
\text { 1) } \tau_{u^{-1}}=\tau_{u} \text {; 2) } \tau_{u v}=\tau_{v u} \text {; 3) } \tau_{v u v^{-1}}=\tau_{u} \text {; 4) } \tau_{u v}=\tau_{u} \tau_{v}-\tau_{u v^{-1}} \text {. }
$$

Следуюшее утверждение достаточно хорошо известно и его легко доказать непосредственньм вычислением, хотя трудно указать точную ссылку.

Лемма 2. Для произвольных $\alpha, \beta, \gamma \in \mathbb{C}$ существуют такие матриць $A, B \in \mathrm{SL}_{2}(\mathbb{C})$, что

$$
\tau_{g}(A, B)=\operatorname{tr} A=\alpha, \quad \tau_{h}(A, B)=\operatorname{tr} B=\beta, \quad \tau_{g h}(A, B)=\operatorname{tr} A B=\gamma
$$

Эта лемма означает, в частности, что характеры $\Phi$ рике $\tau_{g}, \tau_{h}, \tau_{g h}$ алгебраически независимы над $\mathbb{C}$ и, следовательно, многочлен $\Phi$ рике $Q_{w}$ элемента $w$ определен однозначно. Далее, нам нужна явная формула для нахождения многочлена Фрике, полученная в [13]. Чтобы сформулировать этот результат, рассмотрим многочлены $P_{n}(\lambda)$, которые удовлетворяют рекуррентньм соотношениям

$$
P_{n}(\lambda)=\lambda P_{n-1}(\lambda)-P_{n-2}(\lambda)
$$

и начальньм условиям

$$
P_{0}(\lambda)=1, \quad P_{-1}(\lambda)=0
$$

Если $n<0$, положим

$$
P_{n}(\lambda)=-P_{|n|-2}(\lambda)
$$

Степень многочлена $P_{n}(\lambda)$ равна $n$, если $n>0$, и равна $|n|-2$, если $n<0$.

Лемма 3. 1) Многочлен $P_{n}(\lambda), n \geqslant 1$, имеет $n$ нулей, определенных формулой

$$
\lambda_{n, k}=2 \cos \frac{k \pi}{n+1}, \quad k=1,2, \ldots, n .
$$

2) $P_{n-1}(2)=n$ для любого $n \in \mathbb{Z}$.

3) Пусть $x=\tau_{g}, y=\tau_{h}, z=\tau_{g h}$. Тогда

$$
Q_{g h^{n}}(x, y, z)=P_{n-1}(y) z-P_{n-2}(y) x .
$$


ДоКАЗАТЕЛЬСТво. 1) Индукцией по $n$ легко проверить, что

$$
P_{n}(2 \cos \varphi)=\frac{\sin (n+1) \varphi}{\sin \varphi} .
$$

Теперь утверждение п. 1) получается непосредственным вычислением.

Пункты 2) и 3 ) доказываются индукцией по $n$ (по поводу (2) см. также [12, формула (6)]).

Далее, пусть $w=g^{\alpha_{1}} h^{\beta_{1}} \cdots g^{\alpha_{s}} h^{\beta_{s}} \in F_{2}$ - циклически редуцированное слово в $F_{2}$ и пусть $x=\tau_{g}, y=\tau_{h}, z=\tau_{g h}$. Рассмотрим многочлен $\Phi$ рике $Q_{w}(x, y, z)$ как многочлен от $z$. Пусть

$$
Q_{w}(x, y, z)=M_{n}(x, y) z^{n}+M_{n-1}(x, y) z^{n-1}+\cdots+M_{0}(x, y) .
$$

ПРеДЛОЖЕНИЕ 2 (см. [13]). Степень многочлена Фрике $Q_{w}(x, y, z)$ относительно z равна s, т.е. равна числу блоков вида $g^{\alpha_{i}} h^{\beta_{i}}$ в w. Стариий коэффичиент $M_{s}(x, y)$ многочлена $Q_{w}(x, y, z)$ имеет вид:

$$
M_{s}(x, y)=\prod_{i=1}^{s} P_{\alpha_{i}-1}(x) P_{\beta_{i}-1}(y) .
$$

Следуюшая лемма играет важную роль в доказательстве теорем 1 и 2.

Лемма 4. Пусть $G=\left\langle a, b \mid a^{n}=R^{m}(a, b)=1\right\rangle$ u nусть $A, B \in \mathrm{SL}_{2}(\mathbb{C})$ такие матриць, что $\operatorname{tr} A=\alpha$, где $\alpha= \pm 2 \cos (t \pi / n)$ для некоторого $t \in\{1, \ldots, n-1\}$, и $Q_{R}(\alpha, y, z)=c$, где $Q_{R}-$ многочлен Фрике әлемента $R(g, h) \in F_{2}, c= \pm 2 \cos (r \pi / m)$ для некоторого $r \in\{1, \ldots, m-1\}, y=\operatorname{tr} B$, $z=\operatorname{tr} A B$. Положим $H=\langle A, B\rangle$. Предположим, что выполнены следующие два условия:

1) существует унипотентный (либо конечного порядка) әлемент и $\in H$ вида и $=A^{\alpha_{1}} B^{\beta_{1}} \cdots A^{\alpha_{s}} B^{\beta_{s}}$ mакой, что $l=\sum_{i=1}^{s} \beta_{i} \neq 0$;

2) существует әлемент $h \in H$ такой, что $\operatorname{tr} h \notin \mathscr{O}$.

Тогда группа $G$ является нетривиальным амальгамированным свободныцм произведением.

ДокАЗАТЕльство. Покажем, что группа $H$ не удовлетворяет условиям 1)-3) предложения 1. Предположим, что $f: H \rightarrow \mathbb{Z}$ - такой эпиморфизм, что $f(z)=0$ для любого унипотентного элемента $z \in H$. Тогда $f(A)=0$, поскольку $A^{2 n}=E$ в силу леммы 1 . Далее, $f(u)=l f(B)=0$, а значит, $f(B)=0$, поскольку по условию $u$ либо унипотентен, либо имеет конечный порядок. Таким образом, $f(H)=\{0\}-$ противоречие. Далее, по условию сушествует элемент $h \in H$ такой, что $\operatorname{tr} h \notin \mathscr{O}$. Следовательно, $H$ не удовлетворяет условиям 2) и 3 ) предложения 1 . Таким образом, $H$ является нетривиальным амальгамированным свободным произведением, т.е. $H=H_{1} *_{F} H_{2}$, где $H_{1} \neq F \neq H_{2}$. Так как $-E \in Z(H)$, то $-E \in F$. Пусть $\bar{A}, \bar{B}, \bar{H}, \bar{H}_{1}, \bar{H}_{2}, \bar{F}$ являются образами $A, B, H, H_{1}, H_{2}, F$ в $\mathrm{PSL}_{2}(\mathbb{C})$, соответственно. Тогда $\bar{H}_{1} \neq \bar{F} \neq \bar{H}_{2}$ и, следовательно, $\bar{H}=\bar{H}_{1} * \bar{F}_{2}-$ нетривиальное амальгамированное свободное произведение. Условие $Q_{R}(\alpha, y, z)=c$ означает, что $\operatorname{tr} R(A, B)=c$. Тогда в силу леммы $1 R^{2 m}(A, B)=E$. Таким образом, 
$\bar{A}^{n}=R^{m}(\bar{A}, \bar{B})=1$ в $\mathrm{PSL}_{2}(\mathbb{C})$. Следовательно, $\bar{H}$ является эпиморфинцм образом $G$, и мы получаем утверждение леммы 4 . (Хорошо известно, что если $\varphi: \Gamma_{1} \rightarrow \Gamma_{2}-$ эпиморфизм групп и $\Gamma_{2}$ является нетривиальным амальгамированным свободньп произведением, то и $\Gamma_{1}$ такова.)

Следуюшая лемма будет неоднократно использоваться в дальнейшем.

Лемма 5. 1) Для заданных $s, m, M \in \mathbb{Z}$, удовлетворяющих условиям $m \geqslant 2,|M| \geqslant 3$, существуют $\varepsilon \in\{-1,1\}$ и $r \in\{1, \ldots, m-1\}$ такие, что $\left((-1)^{s} 2-c\right) / M \notin \mathscr{O}$, где $c=2 \varepsilon \cos (r \pi / m)$.

2) Для заданного $m \in \mathbb{Z}, m \geqslant 2$, существует $r \in\{1, \ldots, m-1\}$ такое, ито $(2+2 \cos (r \pi / m))^{-1} \notin \mathscr{O}$.

3) Eсли $m \in \mathbb{Z}, m \geqslant 3, m o \cos (\pi / m) \notin \mathscr{O}$.

4) Для любых заданных $m, M \in \mathbb{Z}, m \geqslant 3 u|M| \geqslant 3$, существует такое $r \in\{1, \ldots, m-1\}$, ито $(4 / M) \cos (r \pi / m) \notin \mathscr{O}$.

ДоказАТЕЛЬСТво. 1) Не теряя общности, мы можем считать, что $s$ четно. Предположим, что для любых $\varepsilon \in\{-1,1\}$ и $r \in\{1, \ldots, m-1\}$ число $(2-c) / M$ является целым алгебраическим. Тогда число $(2-c) / M+(2+c) / M=4 / M$ также целое алгебраическое. Так как $|M| \geqslant 3$, это возможно лиш в случае, когда $|M|=4$. Пусть, для определенности, $M=4$. Если $m$ четно, мы положим $r=m / 2$. Тогда $c=0$ и $2 / M=\frac{1}{2} \notin \mathscr{O}$ - противоречие. Пусть $m$ нечетно и пусть $F_{1}=\{2 \cos (2 r \pi / m) \mid r=1, \ldots, m-1\}$. Тогда

$$
\sum_{c \in F_{1}} \frac{2-c}{4}=\frac{2(m-1)-\sum_{c \in F_{1}} c}{4}=\frac{2(m-1)+1}{4},
$$

поскольку $1+\sum_{c \in F_{1}} c=0$ как сумма всех корней из 1 степени $m$. Число $(2(m-1)+1) / 4$, очевидно, не является целым алгебраическим - противоречие, доказывающее 1$)$.

2) Так как

$$
\frac{1}{2+2 \cos (r \pi / m)}=\frac{1}{4 \cos ^{2}(r \pi /(2 m))},
$$

то достаточно доказать, что для некоторого $r \in\{1, \ldots, m-1\}$ число $(2 \cos (r \pi /(2 m)))^{-1}$ не принадлежит $\mathscr{O}$. Согласно лемме 3 многочлен $P_{2 m-1}(\lambda)$ имеет корни $0, \pm 2 \cos (r \pi /(2 m)), r=1, \ldots, m-1$. Легко проверить индукцией по $m$, что

$$
P_{2 m-1}(\lambda)=\lambda\left(\lambda^{2 m-2}+a_{1} \lambda^{2 m-4}+\cdots+(-1)^{m+1} m\right) .
$$

Следовательно, числа $( \pm 2 \cos (r \pi /(2 m)))^{-1}$ являются корнями многочлена $P_{2 m-1}(1 / \lambda)$ или, что эквивалентно, корнями многочлена

$$
g_{1}(\lambda)=\lambda^{2 m-2}+\cdots+(-1)^{m+1} \frac{1}{m}
$$

Так как $1 / m \notin \mathscr{O}$, то по меньшей мере один из корней многочлена $g_{1}(\lambda)$ не является цельм алгебраическим, что и доказывает 2 ). 
3) Достаточно доказать, что сушествует такое $r \in\{1, \ldots, m-1\}$, что $\cos (r \pi / m)$ $\notin \mathscr{O}$, поскольку если $\cos (\pi / m) \in \mathscr{O}$, то и $\cos (r \pi / m) \in \mathscr{O}$ для любого $r \in \mathbb{Z}$. В силу леммы 3 числа $2 \cos (r \pi / m), r=1, \ldots, m-1$, являются корнями многочлена $P_{m-1}(\lambda)$. Если $m=2 k$, то из (3) следует, что числа $\cos (r \pi /(2 k)), r=1, \ldots, 2 k-1$, являются корнями многочлена $g_{1}(2 \lambda)$ или, что эквивалентно, корнями уравнения

$$
\lambda^{2 k-1}+\cdots+(-1)^{k-1} \frac{k}{2^{2 k-2}} \lambda=0
$$

Так как $k \geqslant 2$, то $k<2^{2 k-2}$ и, следовательно, число $k / 2^{2 k-2}$ не является целым алгебраическим. Следовательно, существует $r$ такое, что $\cos (r \pi /(2 k)) \notin \mathscr{O}$.

Пусть $m=2 k+1$. Тогда по индукции легко проверить, что

$$
P_{2 k}(\lambda)=\lambda^{2 k}+\cdots+(-1)^{k}
$$

В силу леммы 3 числа $\cos (r \pi /(2 k+1)), r=1, \ldots, 2 k$, являются корнями многочлена $P_{2 k}(2 \lambda)$ или, что эквивалентно, корнями уравнения

$$
\lambda^{2 k}+\cdots+\frac{(-1)^{k}}{2^{2 k}}=0
$$

Так как $(-1)^{k} / 2^{2 k} \notin \mathscr{O}$, то сушествует $r$ такое, что $\cos (r \pi /(2 k+1)) \notin \mathscr{O}$.

4) Из леммы 3 следует, что числа $(4 / M) \cos (r \pi / m), r=1, \ldots, m-1$, являются корнями многочлена $P_{m-1}(M \lambda / 2)$. Рассмотрим два случая.

Пусть $m=2 k+1$. Тогда в силу (4) числа $(4 / M) \cos (r \pi /(2 k+1)), r=1, \ldots, 2 k$, являются корнями уравнения

$$
\lambda^{2 k}+\cdots+(-1)^{k}\left(\frac{2}{M}\right)^{2 k}=0
$$

Так как $|M| \geqslant 3$, то $(-1)^{k}(2 / M)^{2 k} \notin \mathscr{O}$, следовательно, последнее уравнение имеет корень, который также не принадлежит $\mathscr{O}$.

Пусть $m=2 k$. Тогда в силу (3) числа $(4 / M) \cos (r \pi / m), r=1, \ldots, m-1$, являются корнями уравнения

$$
\lambda^{2 k-1}+\cdots+(-1)^{k+1} k\left(\frac{2}{M}\right)^{2 k-2} \lambda=0 .
$$

Легко видеть, что $(-1)^{k+1} k(2 / M)^{2 k-2} \notin \mathscr{O}$. Тем самым 4$)$, а вместе с тем и лемма 5 доказаны.

\section{§2. Доказательство теоремы 1}

Пусть группа $G$ удовлетворяет условиям теоремы 1 . Если $n>1$, то мы можем рассмотреть групш $G_{1}=\left\langle a, b \mid a^{2}=R^{m}(a, b)=1\right\rangle$, которая является эпиморфным образом $G$. Таким образом, мы можем предположить без потери обшности, что $n=1$. Наша цель состоит в том, чтобы построить представление 
$\varphi: G \rightarrow \mathrm{PSL}_{2}(\mathbb{C})$ такое, что группа $\varphi(G)$ является нетривиальным амальгамированным свободньм произведением. Существуют следующие три случая, каждьй из которых мы рассмотрим отдельно:

1) $R(a, b)=a b^{n_{1}} \cdots a b^{n_{s}}$ является циклически редуцированньм словом, лежащим в подгруппе свободной группы с образующими $a$ и $b$, порожденной $a^{2}$ и коммутантом, и при этом сушествует $i$ такое, что $\left|n_{i}\right|>1$;

2) $R(a, b)$ такое же как в случае 1$)$, при этом $\left|n_{i}\right|=1$ для всех $i=1, \ldots, s$;

3) $R(a, b)$ является циклически редуцированным словом, которое не принадлежит подгруппе свободной группы с образующими $a$ и $b$, порожденной $a^{2}$ и коммутантом.

Случай 1 . Пусть $R(a, b)=a b^{n_{1}} \cdots a b^{n_{s}}$. Из условий, наложенных на элемент $R(a, b)$, следует, что $s$ четно и $\sum_{i=1}^{s} n_{i}=0$. Чтобы применить лемму 4 , покажем, что существуют матрицы $A, B \in \mathrm{SL}_{2}(\mathbb{C})$ такие, что

$$
\operatorname{tr} A=0, \quad \operatorname{tr} B=2, \quad \operatorname{tr} A B=z,
$$

где $z$ - некоторое комплексное число, не принадлежашее $\mathscr{O}$ и удовлетворяюшее уравнению

$$
Q_{R}(0,2, z)=c
$$

$c= \pm 2 \cos (r \pi / m)$ для некоторого $r \in\{1, \ldots, m-1\}$.

Согласно предложению 2 мы можем записать (5) в виде

$$
M_{s}(0,2) z^{s}+M_{s-1}(0,2) z^{s-1}+\cdots+M_{0}(0,2)-c=0,
$$

где $M_{s}(0,2)=\prod_{i=1}^{s} P_{0}(0) P_{n_{i}-1}(2)=\prod_{i=1}^{s} n_{i}$ в силу леммы 3 . Так как $s$ четно, $\sum_{i=1}^{s} n_{i}=0$ и сушествует $i$ такое, что $\left|n_{i}\right|>1$, то $\prod_{i=1}^{s}\left|n_{i}\right| \geqslant 3$. Таким образом, $\left|M_{s}(0,2)\right| \geqslant 3$. Далее, справедлива следуюшая

Лемма 6. Пусть $R=g h^{n_{1}} \cdots g h^{n_{s}} \in F_{2}$, где s четно, и $Q_{R}(x, y, z)=$ $M_{s}(x, y) z^{s}+\cdots+M_{0}(x, y)-$ многочлен Фрике әлемента $R$, где $x=\tau_{g}, y=\tau_{h}$, $z=\tau_{g h}$. Тогда $M_{0}(0,2)=(-1)^{s / 2} 2$.

ДокАЗАТЕльство. Прежде всего, сделаем следующие наблюдения.

1) $Q_{h^{i}}(0,2, z)=2$, поскольку если $A, B \in \mathrm{SL}_{2}(\mathbb{C})$ и $B$ - унипотентная матрица, то $B^{i}$ унипотентна для любого $i$. Следовательно, $\tau_{h^{i}}(A, B)=\operatorname{tr} B^{i}=2$.

2) Из леммы 3 следует, что $Q_{g h^{i}}(0,2, z)=P_{i-1}(2) z=i z$.

Утверждение леммы докажем индукцией по $s$. Если $s=2$, то используя соотношения (1), получаем

$$
Q_{g h^{n_{1}}} g h^{n_{2}}(0,2, z)=Q_{g h^{n_{1}}}(0,2, z) Q_{g h^{n_{2}}}(0,2, z)-Q_{h^{n_{2}-n_{1}}}(0,2, z)=n_{1} n_{2} z^{2}-2
$$

т.е. $M_{0}(0,2)=-2$. Для произвольного $s>2$ имеем

$$
\begin{aligned}
Q_{R}(0,2, z)=Q_{g h^{n_{1}}}(0,2, z) & Q_{g h^{n_{2}} \cdots g h^{n_{s}}}(0,2, z) \\
& -Q_{g h^{n_{3}} \cdots g h^{n_{s}-n_{1}+n_{2}}}(0,2, z)=n_{1} z f(z)-g(z),
\end{aligned}
$$


где

$$
\begin{aligned}
& f(z)=Q_{g h^{n_{2}} \cdots g h^{n_{s}}}(0,2, z), \\
& g(z)=Q_{g h^{n_{3}} \cdots g h^{n_{s}-n_{1}+n_{2}}}(0,2, z) .
\end{aligned}
$$

Мы видим, что свободный член многочлена $Q_{R}(0,2, z)$ совпадает со свободным членом $g(z)$. По индукции имеем

$$
M_{0}(0,2)=-(-1)^{(s-2) / 2} 2=(-1)^{s / 2} 2 .
$$

Лемма 6 доказана.

Учитьвая лемму 6, мы можем записать (6) в виде

$$
z^{s}+\frac{M_{s-1}(0,2)}{M_{s}(0,2)} z^{s-1}+\cdots+\frac{(-1)^{s / 2} 2-c}{M_{s}(0,2)}=0 .
$$

Из 1) леммы 5 следует, что можно выбрать $c= \pm 2 \cos (r \pi / m)$ в уравнении $(7)$ таким, чтобы свободньй коэффициент $\left((-1)^{s / 2}-c\right) / M_{s}(0,2)$ не принадлежал $\mathscr{O}$. Тогда (7) имеет корень $z_{0}$, который не является целым алгебраическим. Согласно лемме 2 существуют матрицы $A, B \in \mathrm{SL}_{2}(\mathbb{C})$ такие, что

$$
\operatorname{tr} A=0, \quad \operatorname{tr} B=2, \quad \operatorname{tr} A B=z_{0} .
$$

Применение леммы 4 завершает доказательство теоремы 1 в первом случае.

Случай 2. Пусть $R(a, b)=a b^{\varepsilon_{1}} a b^{\varepsilon_{2}} \cdots a b^{\varepsilon_{s}}$. Из наших предположений следует, что $s$ четно, $\varepsilon_{i} \in\{-1,1\}$ для всех $i=1, \ldots, s$ и $\sum_{i=1}^{s} \varepsilon_{i}=0$.

Предположим вначале, что для некоторого $j<s$ мы имеем $\varepsilon_{j}=\varepsilon_{j+1}$. Пусть $c=a, d=a b^{\varepsilon_{j}}-$ новые образующие группы $G$. Нетрудно проверить, что тогда $G$ имеет копредставление вида

$$
G=\left\langle c, d \mid c^{2}=R_{1}^{m}(c, d)=1\right\rangle
$$

где $R_{1}(c, d)=c d^{l_{1}} \cdots c d^{l_{t}}$ - циклически редуцированное слово, лежащее в подгруппе свободной групшы с образующими $c$ и $d$, порожденной $c^{2}$ и коммутантом, и при этом сушествует $l_{i}$ такое, что $\left|l_{i}\right| \geqslant 2$. Этот случай был рассмотрен выше в первом случае.

Таким образом, пусть $R(a, b)=a b a b^{-1} \cdots a b a b^{-1}=\left(a b a b^{-1}\right)^{l}$ для некоторого $l>0$. Следовательно, группа $G$ имеет копредставление вида

$$
G=\left\langle a, b \mid a^{2}=\left(a b a b^{-1}\right)^{t}=1\right\rangle
$$

где $t \geqslant 2$. Покажем, что существуют матрищы $A, B \in \mathrm{SL}_{2}(\mathbb{C})$, удовлетворяющие условиям:

1) $\left.\left.\operatorname{tr} A=0 ; 2) \operatorname{tr} A B^{-2} A B^{3}=2 ; 3\right) \operatorname{tr} A B \notin \mathscr{O} ; \quad 4\right) \operatorname{tr} A B A B^{-1}=c$ 
где $c= \pm 2 \cos (r \pi / m)$ для некоторого $r \in\{1, \ldots, m-1\}$. Используя характеры Фрике, условия 2) и 4) можно записать в виде системы:

$$
\left\{\begin{array}{l}
\tau_{g h g h^{-1}}(A, B)=c \\
\tau_{g h^{-2} g h^{3}}(A, B)=2 .
\end{array}\right.
$$

Используя соотношения (1) между характерами Фрике, легко получить, что

$$
\tau_{g h h^{-1}}=-\tau_{g h}^{2}+\tau_{g} \tau_{h} \tau_{g h}-\tau_{h}^{2}+2
$$

и

$$
\tau_{g h^{-2} g h^{3}}=\left(\tau_{g} \tau_{h}^{2}-\tau_{h} \tau_{g h}-\tau_{g}\right)\left(\tau_{h}^{2} \tau_{g h}-\tau_{g} \tau_{h}-\tau_{g h}\right)-\tau_{h}^{5}+5 \tau_{h}^{3}-5 \tau_{h}
$$

Положим $y=\tau_{h}(A, B)=\operatorname{tr} B, z=\tau_{g h}(A, B)=\operatorname{tr} A B$. Так как $\tau_{g}(A, B)=$ $\operatorname{tr} A=0$, мы получаем

$$
\tau_{g h g h^{-1}}(A, B)=-z^{2}-y^{2}+2, \quad \tau_{g h^{-2} g h^{3}}(A, B)=-\left(y^{3}-y\right) z^{2}-\left(y^{5}-5 y^{3}+5 y\right) .
$$

Таким образом, (8) можно записать в виде:

$$
\left\{\begin{array}{l}
z^{2}+y^{2}-2+c=0 \\
\left(y^{3}-y\right) z^{2}+y^{5}-5 y^{3}+5 y+2=0
\end{array}\right.
$$

Из (9) следует, что

$$
y^{3}-\left(1+\frac{1}{c+2}\right) y-\frac{2}{c+2}=0 .
$$

Согласно 2) леммы 5 существует такое $r \in\{1, \ldots, m-1\}$, что число $1 /(c+2)$ не принадлежит $\mathscr{O}$. Тогда уравнение $(10)$ имеет корень $y_{0} \notin \mathscr{O}$. Пусть $\left(y_{0}, z_{0}\right)-$ некоторое решение (9). Согласно лемме 2 сушествуют матрищы $A, B \in \mathrm{SL}_{2}(\mathbb{C})$ такие, что

$$
\operatorname{tr} A=0, \quad \operatorname{tr} B=y_{0}, \quad \operatorname{tr} A B=z_{0} .
$$

Применяя лемму 4, завершаем доказательство теоремы 2 во втором случае.

Случай 3. Пусть $R(a, b)=a b^{n_{1}} \cdots a b^{n_{s}}$ и $R(a, b)$ не принадлежит подгрупше свободной группы с образуюшими $a$ и $b$, порожденной $a^{2}$ и коммутантом. Случай $\sum_{i=1}^{s} n_{i} \neq 0$ был рассмотрен в [2, теорема 5]. Поэтому мы можем считать, что $s$ нечетно и $\sum_{i=1}^{s} n_{i}=0$.

Вначале рассмотрим случай $m=2$. Пусть $G_{1}=\left\langle a, b \mid a^{2}=b^{2}=R^{2}(a, b)=1\right\rangle$. Покажем, что $G_{1}=\left\langle a, b \mid a^{2}=b^{2}=1\right\rangle$ является свободным произведением двух циклических групп второго порядка. Достаточно доказать, что $R^{2}(a, b)=1$ в $G$. Мы докажем более общий факт: если $w(a, b)=a b^{l_{1}} \cdots a b^{l_{s}}$, где $s=2 k+1$ и $\sum_{i=1}^{s} l_{s}$ - четное число, тогда $w^{2}(a, b)=1$ в $G_{1}$. Воспользуемся индукцией по $s$. Если $s=1$, то утверждение очевидно. Для произвольного $s$ среди показателей $l_{1}, \ldots, l_{s}$ существует четный. Пусть, например, $l_{1}$ четно. Тогда $b^{l_{1}}=1$ в $G_{1}$ и

$$
w(a, b)=b^{l_{2}} a b^{l_{3}} \cdots a b^{l_{s}}
$$


т.е. $w(a, b)$ сопряжено с

$$
w_{1}(a, b)=a b^{l_{3}} \cdots a b^{l_{2}+l_{s}} .
$$

Поскольку сумма $l_{3}+\cdots+\left(l_{2}+l_{s}\right)$ по-прежнему четна, мы можем применить индукцию и получить, что $w_{1}^{2}(a, b)=1$ в $G_{1}$. Следовательно, $w^{2}(a, b)=1$ в $G_{1}$. Поскольку группа $G_{1}$ является эпиморфным образом $G$, мы получаем, что $G$ является нетривиальным амальгамированньгм свободньм произведением.

Рассмотрим теперь случай $m \geqslant 3$. Покажем, что сушествуют такие матрицы $A, B \in \mathrm{SL}_{2}(\mathbb{C})$, что

$$
\operatorname{tr} A=0, \quad \operatorname{tr} B=y_{0}, \quad \operatorname{tr} A B=2, \quad Q_{R}\left(0, y_{0}, 2\right)=2 \cos \frac{\pi}{m},
$$

где $y_{0}$ - некоторое комплексное число, не являющееся целым алгебраическим и $Q_{R}(x, y, z)$ - многочлен $\Phi$ рике слова $R(g, h) \in F_{2}$. Справедлива

ЛЕмма 7. 1) Справедливо следующее равенство: $Q_{R}(0, y, z)=z f(y, z)$, әде $f(y, z) \in \mathbb{Z}[y, z]$.

2) Многочлен $f_{1}(y)=f(y, 2)$ не является константой.

ДокаЗАТЕЛЬство. 1) Из леммы 3 следует, что $Q_{g h^{n}}(0, y, z)=P_{n-1}(y) z$ и при $s=1$ утверждение доказано. Пусть $s>1$. Тогда, используя соотношения (1), получаем

$$
\tau_{g h^{n_{1}} \cdots g h^{n_{s}}}=\tau_{g h^{n_{1}}} \tau_{g h^{n_{2}} \cdots g h^{n_{s}}}-\tau_{g h^{n_{3}} \cdots g h^{n_{s}-n_{1}+n_{2}}} .
$$

По индукции имеем

$$
Q_{R}(0, y, z)=P_{n_{1}-1}(y) f_{1}(y, z) z-z f_{2}(y, z)=z f(y, z)
$$

для некоторого многочлена $f(y, z) \in \mathbb{Z}[y, z]$.

2 ) Рассмотрим $\mathbb{Z}$-алгебру $T$, порожденную всеми характерами $\Phi$ рике $\tau_{w}$, $w \in F_{2}$. Любой автоморфизм $\sigma \in \operatorname{Aut}\left(F_{2}\right)$ индуцирует автоморфизм

$$
\sigma^{\prime}: T \rightarrow T, \quad \tau_{w} \mapsto \tau_{\sigma(w)}
$$

Рассмотрим автоморфизм $\sigma \in \operatorname{Aut}\left(F_{2}\right)$ такой, что

$$
\sigma(g)=g, \quad \sigma(h)=g h
$$

Тогда $\sigma^{\prime} \in \operatorname{Aut}(T)$ такой автоморфизм, что

$$
\sigma^{\prime}(x)=x_{1}=x, \quad \sigma^{\prime}(y)=y_{1}=z, \quad \sigma^{\prime}(z)=z_{1}=x z-y
$$

Далее, имеем

$$
\sigma^{\prime}\left(\tau_{R(g, h)}\right)=\tau_{\sigma(R(g, h))}=\tau_{R(\sigma(g), \sigma(h))},
$$

следовательно,

$$
\sigma^{\prime}\left(Q_{R}(x, y, z)\right)=Q_{R}\left(x_{1}, y_{1}, z_{1}\right)=Q_{R}(x, z, x z-y)
$$


Таким образом, $\sigma^{\prime}\left(Q_{R}(0, y, 2)\right)=Q_{R}(0,2,-y)$. Из предложения 2 следует, что многочлен $Q_{R}(0,2,-y) \in \mathbb{Z}[y]$ имеет степень $s$, поскольку для его старшего коэффициента $M_{s}$ вьполнено $\left|M_{s}\right|=\prod_{i=1}^{s}\left|n_{i}\right| \neq 0$. Итак, $Q_{R}(0,2,-y)$ - не константа, следовательно, $\sigma^{\prime-1}\left(Q_{R}(0,2,-y)\right)=Q_{R}(0, y, 2)=2 f_{1}(y)$ - также не константа. Лемма 7 доказана.

Теперь мы можем закончить доказательство теоремы 1 . Согласно лемме 7 равенство $Q_{R}\left(0, y_{0}, 2\right)=2 \cos (\pi / m)$ эквивалентно равенству

$$
f_{1}\left(y_{0}\right)=\cos \frac{\pi}{m} .
$$

В силу 3$)$ леммы 5 число $\cos (\pi / m)$ не является целым алгебраическим, следовательно, существует корень $y_{0}$ уравнения (11), которьй также не является целым алгебраическим. В силу леммы 2 существуют матрицы $A, B \in \mathrm{SL}_{2}(\mathbb{C})$ такие, что

$$
\operatorname{tr} A=0, \quad \operatorname{tr} B=y_{0}, \quad \operatorname{tr} A B=2 .
$$

Применив лемму 4, мы завершаем доказательство теоремы 1.

\section{§3. Доказательство теоремы 2}

Учитьвая теорему 1 , мы можем считать, что $n \geqslant 3$. Пусть $R(g, h) \in F_{2}$ и пусть $Q_{R}$ - многочлен $\Phi$ рике слова $R$. Покажем, что сушествуют матрицы такие $A, B \in \mathrm{SL}_{2}(\mathbb{C})$, что

$$
\operatorname{tr} A=\alpha, \quad \operatorname{tr} B=2, \quad \operatorname{tr} A B=z,
$$

где $\alpha=2 \cos (\pi / n), z$ - некоторое комплексное число, не являюшееся целым алгебраическим и удовлетворяюшее уравнению

$$
Q_{R}(\alpha, 2, z)=c
$$

где $c= \pm 2 \cos (r \pi / m)$ для некоторого $r \in\{1, \ldots, m-1\}$. Согласно предложению 2 мы можем записать (12) в виде

$$
M_{s}(\alpha, 2) z^{s}+M_{s-1}(\alpha, 2) z^{s-1}+\cdots+M_{0}(\alpha, 2)-c=0
$$

где

$$
M_{s}(\alpha, 2)=\prod_{i=1}^{s} P_{u_{i}-1}(\alpha) P_{v_{i}-1}(2) .
$$

В силу леммы $3 P_{u_{i}-1}(\alpha) \neq 0, P_{v_{i}-1}(2)=v_{i}$, поэтому $M_{s}(\alpha, 2) \neq 0$. Запишем $(13)$ в виде

$$
z^{s}+\frac{M_{s-1}(\alpha, 2)}{M_{s}(\alpha, 2)}+\cdots+\frac{M_{0}(\alpha, 2)-c}{M_{s}(\alpha, 2)}=0 .
$$

Покажем, что мы можем выбрать $c$ так, что $\left(M_{0}(\alpha, 2)-c\right) / M_{s}(\alpha, 2) \notin \mathscr{O}$. Предположим, что для любого $r \in\{1, \ldots, m-1\}$ оба числа

$$
\frac{M_{0}(\alpha, 2)+2 \cos (r \pi / m)}{M_{s}(\alpha, 2)} \quad \text { и } \quad \frac{M_{0}(\alpha, 2)-2 \cos (r \pi / m)}{M_{s}(\alpha, 2)}
$$


принадлежат $\mathscr{O}$. Тогда их разность

$$
\frac{4 \cos (r \pi / m)}{M_{s}(\alpha, 2)} \in \mathscr{O}
$$

Так как для любого $r$ многочлен $P_{r}(\lambda)$ имеет целые коэффициенты, то $P_{u_{i}-1}(\alpha) \in \mathscr{O}$. Следовательно, для любого $r \in\{1, \ldots, m-1\}$

$$
\frac{4 \cos (r \pi / m)}{M_{s}(\alpha, 2)} P_{u_{i}-1}(\alpha)=\frac{4 \cos (r \pi / m)}{M} \in \mathscr{O},
$$

где $M=\prod_{i=1}^{s} v_{i} \in \mathbb{Z}$ и $|M| \geqslant 3$ по условию теоремы. Мы получили противоречие c 4) леммы 5 . Это означает, что существует корень $z_{0}$ уравнения (14), который не принадлежит $\mathscr{O}$. В силу леммы 2 сушествуют матрицы $A, B \in \mathrm{SL}_{2}(\mathbb{C})$ такие, что

$$
\operatorname{tr} A=\alpha, \quad \operatorname{tr} B=2, \quad \operatorname{tr} A B=z_{0} .
$$

Применив лемму 4, мы завершаем доказательство теоремы 2.

В заключение сформулируем следуюшую гипотезу.

ГипотезА 2. Группа $G=\left\langle a, b \mid a^{n}=R^{m}(a, b)=1\right\rangle$, әде $m \geqslant 2, n=0$ или $n \geqslant 2$ и $R(a, b)$ - ииклически редуцированное слово в свободной группе с образующими а и $b$, содержсаее $b$, является нетривиальным амальгамированнымм свободным произведением.

Учитьвая теоремы 1,2 и теорему 5 из [2], эта гипотеза не доказана в следующем случае: $n$ - нечетно, $R(a, b)=a^{l} R_{1}(a, b)$, где $0 \leqslant l<n$ и $R_{1}(a, b)=$ $a^{u_{1}} b^{v_{1}} \cdots a^{u_{s}} b^{v_{s}}$ - циклически редуцированное слово, лежашее в коммутанте свободной групшы с образуюшими $a$ и $b$, такое, что $\prod_{i=1}^{s}\left|v_{i}\right| \in\{1,2\}$.

Эта статья была написана во время посещения автором университета г. Билефельд (ФРГ) в качестве гостя SFB 343 "Diskrete Strukturen in der Mathematik".

\section{Список литературы}

1. Howie J. One relator products of groups // Proceedings of groups St. Andrews. Cambridge: Cambridge Univ. Press, 1985. P. 216-220.

2. Fine B., Levin F., Rosenberger G. Free subgroups and decompositions of one-relator products of cyclics; Part 2: Normal torsion free subgroups and FPA decompositiomns // J. Indian Math. Soc. 1985. V. 49. P. 237-247.

3. Zieschang H. On decompositions of discontinuous groups of the plane // Math. Z. 1976. V. 151. P. 165-188.

4. Rosenberger G. Bemerkungen zu einer Arbeit von H. Zieschang // Arch. Math. (Basel). 1977. V. 29. P. 623-627.

5. Long D. D., Maclachlan C., Reid A. W. Splitting groups of signature $(1, n) / /$ J. Algebra. 1996. V. 185. P. 329-341.

6. Dunwoody M. J., Sageev M. Splittings of certain Fuchsian groups // Proc. Amer. Math. Soc. 1997. V. 125. № 7. P. 1953-1954.

7. Магнус B., Каррас А., Солитэр Д. Комбинаторная теория групп. М.: Наука, 1974.

8. Bass $H$. Finitely generated subgroups of $\mathrm{GL}_{2}(\mathbb{C})_{2}(\mathbb{C}) / /$ The Smith Conjecture. New York: Wiley, 1984. P. 127-136. 
9. Horowitz $R$. Characters of free groups represented in the two dimensional linear group // Comm. Pure. Appl. Math. 1972. V. 25. P. 635-649.

10. Magnus $W$. The uses of 2 by 2 matrices in combinatorial group theory // Results Math. 1981. V. 4. № 2. P. 171-192.

11. Culler M., Shalen P. Varieties of group representations and splittings of 3 manifolds // Ann. of Math. (2). 1983. V. 117. P. 109-147.

12. Helling $H$. Diskrete Untergruppen von $\mathrm{SL}_{2}(\mathbb{R}) / /$ Invent. Math. 1972. V. 17. P. 217-229.

13. Traina $C$. Trace polynomial for two generated subgroups of $\mathrm{SL}_{2}(\mathbb{C}) / /$ Proc. Amer. Math. Soc. 1980. V. 79. P. 369-372.

Институт математики НАН Беларуси, Минск

E-mail: benyash@im.bas-net.by
Поступила в редакцию 21.10.1997 